To the Editors:

\title{
Pre and post operative assessment of voice changes in patients undergoing total thyroidectomy
}

\author{
D M C D Dissanayake ${ }^{1}$, R Fernando ${ }^{1}$, B N L Munasinge ${ }^{1}$, S B Thilakarathne ${ }^{1}$, D P Pinto ${ }^{1}$, U A Uragoda ${ }^{1}$
}

Ceylon Medical Journal 2017; 62 115-116

DOI: http://doi.org/10.4038/cmj.v62i2.8481

A change in voice following total thyroidectomy (TT) is a complication feared by many patients. The close proximity of the recurrent laryngeal nerve (RLN) and external branch of superior laryngeal nerve (EBSLN) to the thyroid gland may cause injury to the nerves due to intra operative; traction, indirect thermal injury, their handling, surrounding tissue oedema, as well as haemaoma formation following surgery [1]. In experienced hands this complication occurs in 1-2\%. Thus, fear of nerve injury is a real concern to patient [2]. People who depend on the voice for their professions such as teachers, singers are the most affected.

Strategies such as intra-operative nerve monitoring (IONM) does not seem to help in reducing this complication in routine total thyroidectomy. A voice assessment after TT is not routinely done in an objective manner and the usual post-operative procedure is an indirect laryngeoscopy, which assesses the integrity of RLN. This is clearly inadequate because voice dysfunction is more complex than simply nerve integrity. All components of voice such as; spectrum, pitch, intensity, formant and pulses can be affect in varying degree if there is any injury to RLN, EBSLN or both.

There are only a few articles which have reported an objective assessment of voice. Our study was designed to objectively asses the voice in patients before and after thyroidectomy using a cheap and easily available method using a voice recorder and computer software. The main objective of this study was to assess voice changes after TT in patients with thyroid disease and to evaluate which component of the voice is mainly affected.

All components of voice such as; Pitch, Intensity, Formant and Pulses may be affected in varying degrees with injury to RLN and EBSLN or both. The main components of voice which affects subjective variation of voice are deemed to be intensity and pitch. A prospective cohort study was undertaken to objectively evaluate the intensity and pitch of voice following thyroidectomy and to assess the pattern of recovery of the voice changes.

Fifty four consecutive patients who underwent TT in the Professorial Surgical Unit, Ragama, from September 2015 were studied. All procedures were done by a single consultant surgeon. Equipment used for assessment of voice was a standard microphone and voice recorder. All patients were asked to pronounce vowels ("a, e, i, o, u") in the same order and the word ("Uswatakeiyawa”) during a pre recording rehearsal. All recordings were limited to 6 seconds to simplify the analysis. Same vowels and the word was recorded before and after thyroidectomy. Same file format, venue (calm isolated room) and position (sitting) of the patient was used to minimize errors. Later, the voice was serially assessed using the same equipment after two weeks, six weeks, three months and six months post-operatively. Recorded data were analyzed using standard voice analyzing open source software "PRAAT" which provides analyses of pitch and intensity. Using PRATT the numerical values of minimum, maximum and mean can be extracted, for pitch in hertz $(\mathrm{Hz})$ and intensity in decibels $(\mathrm{dB})$. Pre-operative and post-operative values were used for objective assessment of voice and statistical analysis was done with SPSS version 20 . Careful intra-operative identification of nerves and the lack of clinically noticeable voice change, precluded the need for vocal cord assessment post-operatively.

According to some studies the commonest cause of voice change appears to be injury to the EBSLN on one or both sides [3, 4]. Damage to the RLN, which was routinely identified and protected, was rarely a cause.

${ }^{1}$ Faculty of Medicine, University of Kelaniya, Ragama, Sri Lanka.

Correspondence: CDD, e-mail:<dmcduminda@yahoo.com>. Received 20 January 2017 and revised version accepted 18 April 2017

This is an open-access article distributed under the terms of the Creative Commons Attribution License, which permits unrestricted use, distribution, and reproduction in any medium, provided the original author and source are credited. 
When the EBSLNs were identified and preserved, permanent voice changes occurred in only 5\% [3].

In this study, 8 (14.8\%) patients failed to achieve pre-operative pitch at two weeks. The pitch improved in two out of eight patients after six weeks. In contrast, 34 (62.9\%) patients had low-voice intensity at two weeks, but 26 of them showed significant improvement at six weeks. This indicates that the recovery from possible neuroprexia of the RLN is much quicker than the recovery of EBSLN. This has not been reported in previous studies and is an area which needs further study involving a larger number of patients. All patients were able to achieve preoperative intensity and pitch in three months, which indicates that there was no objectively demonstrable permanent injury to the EBSLN nor RLN. These findings have not been recorded in previous studies.

Almost all patients will have voice issues, though not clinically detectable, after thyroidectomy. In routine practice, only the patients with clinically noticeable voice changes will be referred for laryngoscopic assessment of vocal fold mobility [5]. Guidelines alludes to the inaccuracy of vocal cord movement in assessing voice. All patients in this study underwent pre-operative vocal cord assessment as it is the protocol of the unit. However, as none of the patients had clinically noticeable voice problems, they were not referred for vocal cord assessment post-operatively. Even then, as this study highlights, there can be changes in certain aspects of voice (intensity and pitch) which tends to resolve spontaneously over a period of six weeks.

The main limitations of this study were the small number of patients, not evaluating other components of voice, no data regarding occupation of the patients not carrying out laryngoscopy to evaluate vocal cords postoperatively and not excluding other factors such as sore throat and respiratory tract infections which could affect voice quality.

\section{References}

1. Friedman M, LoSavio P, Ibrahim H. Superior laryngeal nerve identification and preservation in thyroidectomy. Arch. Otolaryngol. Head Neck Surg 2002; 128: 296-303.

2. Shindo M, Chheda NN. Incidence of vocal cord paralysis with and without recurrent laryngeal nerve monitoring during thyroidectomy. Arch Otolaryngol Head Neck Surg 2007; 133: 481-5.

3. Aluffi P, Policarpo M, Cherovac C, Olina M, Dosdegani R, Pia F. Post-thyroidectomy superior laryngeal nerve injury. Eur Arch Otorhinolaryngol 2001; 258: 451-4.

4. Sung WK, Seung TK, Hyo SP, Hyoung SL, Jong CH, Soon BK, Kang DL. Voice examination in patients with decreased high pitch after thyroidectomy. Indian J Otolaryngol Head Neck Surg 2012; 64: 120-30.

5. Chandrasekhar SS, Randolph GW, Seidman MD, et al. Clinical practice guideline: improving voice outcomes after thyroid surgery. Arch Otolaryngol Head Neck Surg 2013; 148: S1-37. 